\title{
Correction: Halofuginone inhibits radiotherapy-induced epithelial-mesenchymal transition in lung cancer
}

\author{
Yang Chen ${ }^{1, *}$, Weishuai Liu ${ }^{2,}{ }^{*}$, Peng Wang ${ }^{3}$, Hailing Hou ${ }^{1}$, Ningbo Liu ${ }^{1}$, Linlin Gong ${ }^{1}$, \\ Youyou Wang ${ }^{1}$, Kai $\mathrm{Ji}^{2}$, Lujun Zhao ${ }^{1}$ and Ping Wang ${ }^{1}$ \\ ${ }^{1}$ Department of Radiation Oncology, Tianjin Medical University Cancer Institute and Hospital, National Clinical Research \\ Center of Cancer, Key Laboratory of Cancer Prevention and Therapy, Tianjin 300060, China \\ 2 Department of Pain Management, Tianjin Medical University Cancer Institute and Hospital, National Clinical Research, \\ Center of Cancer, Key Laboratory of Cancer Prevention and Therapy, Tianjin 300060, China \\ ${ }^{3}$ Department of Radiation Oncology, Peking University International Hospital, Beijing 102206, China \\ * These authors have contributed equally to this work
}

Published: August 03, 2018

Copyright: Chen et al. This is an open-access article distributed under the terms of the Creative Commons Attribution License 3.0 (CC BY 3.0), which permits unrestricted use, distribution, and reproduction in any medium, provided the original author and source are credited.

This article has been corrected: The correct author affiliation is given below:

${ }^{1}$ Department of Radiation Oncology, Tianjin Medical University Cancer Institute and Hospital, National Clinical Research Center of Cancer, Key Laboratory of Cancer Prevention and Therapy, Tianjin 300060, China

Original article: Oncotarget. 2016; 7:71341-71352. https://doi.org/10.18632/oncotarget.11217 